\title{
A C G
}

publications

Org. Commun. 12:4 (2019) 217-221

organic communications

\section{Oxidation of some benzyl substituted fused quinazoline derivatives}

\author{
Dmytro Kravtsov \\ Department of Organic and Bioorganic Chemistry, Zaporizhzhia State Medical University, \\ Mayakovsky ave., 26, 69035, Zaporizhzhia, Ukraine
}

(Received November 04, 2019; Revised December 11, 2019; Accepted December 12, 2019)

\begin{abstract}
In this study, syntheses of some benzoyl substituted fused quinazoline derivatives 4a-d, using Fieser's reagent, are reported. An unexpected product 5a and $\mathbf{6}$ were isolated from the reaction mixture. Based on the experimental data, a possible oxidation mechanism of ketone $4 \mathbf{a}$ with chromium trioxide is described. The synthesized compounds were characterized by ${ }^{1} \mathrm{H},{ }^{13} \mathrm{C},{ }^{19} \mathrm{~F}$ NMR and LC-MS data.
\end{abstract}

Keywords: Oxidation; fused quinazoline derivatives; Fieser's reagent; benzyl to benzoyl; mechanism. (C) 2019 ACG Publications. All rights reserved.

\section{Introduction}

Oxidation reactions are important tools in organic chemistry. This type of processes is particularly attractive for its versatile possibilities. It allows both to modify the molecule by introducing a new hydrophilic group and to carry out its degradation. Today, practically for each reaction the exact or approximate mechanism is known. Based on this, we can easily predict the reaction product.

Herein, an unusual oxidation of benzyl group is described and tested on other samples.

\section{Background}

Fused quinazoline derivatives were frequently described as a potential class of biologically active agents. ${ }^{1-7}$ Thus, the chemical modifications of such compound are highly desirable.

Commonly used method for chemical modification is an oxidation reaction. Fieser's reagent ${ }^{8}$ (a mixture of chromium trioxide in acetic acid) is one of the most accessible and effective oxidation system. ${ }^{9,10}$ The aim of this research is to convert benzyl substituted fused quinazolines to the corresponding benzoyl-containing derivatives using chromium trioxide, during which an unexpected reaction took place.

\section{Present Study}

Chemical Material and Apparatus: Substances 1a-d were synthesized according to the reported procedures. ${ }^{11-13}$ Other chemicals and solvents were obtained from commercially available sources and

\footnotetext{
*Corresponding author: E-mail: kravtsovsynthesis@gmail.com

The article was published by ACG Publications 
were used without additional purification. Melting points were determined in open capillary tubes and uncorrected. The reactions were monitored via silica gel coated aluminum TLC plate, with flourescent indicator $\mathrm{F}_{254}$ (Merck). Column chromatography was carried out using Kieselgel Merck 60 (230-400 mesh) as the stationary phase. ${ }^{1} \mathrm{H},{ }^{13} \mathrm{C}$ and ${ }^{19} \mathrm{~F}$ NMR spectra were recorded at 500 or $400 \mathrm{MHz}, 125$ $\mathrm{MHz}$ and $376 \mathrm{MHz}$, respectively. Chemical shifts were reported in ppm downfield from tetramethylsilane $\left({ }^{1} \mathrm{H},{ }^{13} \mathrm{C}\right)$ or $\mathrm{CFCl}_{3}\left({ }^{19} \mathrm{~F}\right)$, which were used as internal standards. Mass spectra were recorded with an LC-MS instrument using chemical ionization (CI). LC-MS data were acquired with an Agilent 1200 HPLS system equipped with a DAD/ELSD/LCMS-6120 diode matrix and massselective detector, column: Poroshell $120 \mathrm{SBC} 18,4.6 \mathrm{~mm} \times 30 \mathrm{~mm}$; eluent A: acetonitrile/water, 99:1, with $0.1 \%$ of FA; eluent B: water with $0.1 \%$ of FA.

General Procedure: A mixture of the phenylacetic acid (4.4 mmol), CDI (4.8 mmol) and dioxane (20 $\mathrm{mL})$ was stirred at $80{ }^{\circ} \mathrm{C}$ for $1 \mathrm{~h}$. Then, the appropriate amine 1a-d $(4.4 \mathrm{mmol})$ was added and the mixture was refluxed for $3 \mathrm{~h}$., after which $\mathrm{H}_{2} \mathrm{O}(75 \mathrm{~mL})$ was added. The precipitate was filtered, ${ }^{\mathrm{a}}$ washed thoroughly with $\mathrm{H}_{2} \mathrm{O}$ and dried at $60{ }^{\circ} \mathrm{C}$. The crude product was dissolved in $\mathrm{AcOH}(50 \mathrm{~mL})$ and refluxed for $8 \mathrm{~h}$. After that, the solvent was removed in vacuo and $\mathrm{MeOH}(10 \mathrm{~mL})$ was added. The resulting precipitate was filtered and washed with cold $\mathrm{MeOH}$ and dried at $60{ }^{\circ} \mathrm{C} .{ }^{\mathrm{b}} \mathrm{A}$ solution of $\mathrm{CrO}_{3}$ (4.2 $\mathrm{mmol}$ ( three-fold excess)) in $\mathrm{AcOH}(40 \mathrm{~mL})$ was added to a stirred solution of the crude product in $\mathrm{AcOH}(20 \mathrm{~mL})$ over $30 \mathrm{~min}$ at $60-63{ }^{\circ} \mathrm{C}$. The mixture was stirred for $3.5 \mathrm{~h}$. at $60-63{ }^{\circ} \mathrm{C}$, then, poured into a solution of $\mathrm{Na}_{2} \mathrm{SO}_{3}(2.8 \mathrm{mmol})$ in ice-water $(100 \mathrm{~mL})$. The residue was filtered, ${ }^{\mathrm{c}}$ washed thoroughly with $\mathrm{H}_{2} \mathrm{O}$ and dried at $60{ }^{\circ} \mathrm{C}$. The resulting material was purified to obtain the products (silica gel column chromatography EtOAc- $\mathrm{CHCl}_{3}-\mathrm{PE}(6: 2: 2) \mathbf{4 a}^{\mathrm{d}}$ and $\mathbf{4 b}$. Crystallization from DMF$\mathrm{H}_{2} \mathrm{O}$ afforded $\mathbf{4 c}$ and $\mathbf{4 d}$ ). (Please see the supporting information file to see the information of ${ }^{\mathrm{a}-\mathrm{d}}$ )

$N$-(2-(1H-Benzo[d]imidazol-2-yl)phenyl)-2-phenylacetamide (2a): Gray solid. M.p. $211{ }^{\circ} \mathrm{C}$. Yield: 88.9\%. ${ }^{1} \mathrm{H}$ NMR ( $\delta$, ppm, $400 \mathrm{MHz}, \mathrm{DMSO}_{6}$ ): $\delta 13.24$ (s, $\left.1 \mathrm{H}\right), 12.92(\mathrm{~s}, 1 \mathrm{H}), 8.68(\mathrm{~d}, J=8.3 \mathrm{~Hz}$, $1 \mathrm{H}), 8.06(\mathrm{~d}, J=7.8 \mathrm{~Hz}, 1 \mathrm{H}), 7.71(\mathrm{~d}, J=5.6 \mathrm{~Hz}, 1 \mathrm{H}), 7.54(\mathrm{~d}, J=6.0 \mathrm{~Hz}, 1 \mathrm{H}), 7.47(\mathrm{~d}, J=7.1 \mathrm{~Hz}$, 2H), $7.39(\mathrm{t}, J=7.8 \mathrm{~Hz}, 1 \mathrm{H}), 7.31(\mathrm{t}, J=7.3 \mathrm{~Hz}, 2 \mathrm{H}), 7.27-7.20(\mathrm{~m}, 3 \mathrm{H}), 7.17(\mathrm{t}, \mathrm{J}=7.6 \mathrm{~Hz}, 1 \mathrm{H})$, $3.81(\mathrm{~s}, 2 \mathrm{H}) .{ }^{13} \mathrm{C}$ NMR $\left(\delta, \mathrm{ppm}, 125 \mathrm{MHz}, \mathrm{DMSO}-\mathrm{d}_{6}\right): 170.09,153.03,151.15,148.73,138.76$, $135.88,131.08,130.87,129.87,129.00,127.74,127.32,123.36,120.37,116.60,115.89,115.45$, 110.56, 45.90. MS (CI): $\mathrm{m} / z 328.0[\mathrm{M}+\mathrm{H}]^{+}$.

6-Benzylbenzo[4,5]imidazo[1,2-c]quinazoline (3a): White solid. M.p. $199{ }^{\circ} \mathrm{C}$ (lit. 200-202 ${ }^{\circ} \mathrm{C}$ ).$^{14}$ Yield: $70.0 \% .{ }^{1} \mathrm{H}$ NMR $\left(\delta\right.$, ppm, $400 \mathrm{MHz}$, DMSO $\left.\mathrm{d}_{6}\right): 8.58(\mathrm{~d}, J=7.9 \mathrm{~Hz}, 1 \mathrm{H}), 8.10(\mathrm{~d}, J=8.4 \mathrm{~Hz}$, $1 \mathrm{H}), 7.94(\mathrm{~d}, J=8.1 \mathrm{~Hz}, 1 \mathrm{H}), 7.87-7.77(\mathrm{~m}, 2 \mathrm{H}), 7.71(\mathrm{t}, J=7.3 \mathrm{~Hz}, 1 \mathrm{H}), 7.52(\mathrm{t}, J=7.7 \mathrm{~Hz}, 1 \mathrm{H})$, $7.47-7.28(\mathrm{~m}, 5 \mathrm{H}), 7.25(\mathrm{t}, J=6.7 \mathrm{~Hz}, 1 \mathrm{H}), 4.92(\mathrm{~s}, 2 \mathrm{H}) .{ }^{13} \mathrm{C}$ NMR $\left(\delta, \mathrm{ppm}, 125 \mathrm{MHz}, \mathrm{DMSO}-\mathrm{d}_{6}\right)$ : $149.96,147.74,144.31,142.13,135.54,132.34,129.30,129.13,129.04,128.40,127.93,127.29$, $125.79,124.18,123.36,119.96,118.26,115.87,41.53 . \mathrm{MS}(\mathrm{CI}): \mathrm{m} / \mathrm{z} 310.2[\mathrm{M}+\mathrm{H}]^{+}$.

Benzo[4,5]imidazo[1,2-c]quinazolin-6-yl(phenyl)methanone (4a): Yellow solid. M.p. 183-185 ${ }^{\circ} \mathrm{C}$ (lit. 222-224 $\left.{ }^{\circ} \mathrm{C}\right) .{ }^{15}$ Yield: $22.8 \%$. ${ }^{1} \mathrm{H}$ NMR $\left(\delta\right.$, ppm, $\left.400 \mathrm{MHz}, \mathrm{DMSO}-\mathrm{d}_{6}\right): 8.67(\mathrm{~d}, J=7.6 \mathrm{~Hz}, 1 \mathrm{H}), 8.28$ $(\mathrm{d}, J=7.2 \mathrm{~Hz}, 2 \mathrm{H}), 7.99(\mathrm{~d}, J=7.9 \mathrm{~Hz}, 2 \mathrm{H}), 7.89(\mathrm{t}, J=7.3 \mathrm{~Hz}, 1 \mathrm{H}), 7.86-7.79(\mathrm{~m}, 2 \mathrm{H}), 7.65(\mathrm{t}, J=$ $7.6 \mathrm{~Hz}, 2 \mathrm{H}), 7.54(\mathrm{t}, J=7.5 \mathrm{~Hz}, 1 \mathrm{H}), 7.44(\mathrm{~d}, J=8.1 \mathrm{~Hz}, 1 \mathrm{H}), 7.36(\mathrm{t}, J=7.3 \mathrm{~Hz}, 1 \mathrm{H}) .{ }^{13} \mathrm{C} \mathrm{NMR}(\delta$, ppm, $125 \mathrm{MHz}$, DMSO-d $\left.{ }_{6}\right): 188.22$, 147.22, 144.30, 143.88, 141.12, 136.28, 134.00, 132.65, 131.49, $129.99,129.79,128.77,128.00,126.32,124.44,123.74,120.40,119.57,113.83$. MS (CI): $\mathrm{m} / \mathrm{z} 324.0$ $[M+\mathrm{H}]^{+}$.

(2-(4-Fluorophenyl)-[1,2,4]triazolo[1,5-c]quinazolin-5-yl)(phenyl)methanone (4b): White solid. M.p. 233-236 ${ }^{\circ} \mathrm{C}$. Yield: 5.8\%. ${ }^{1} \mathrm{H}$ NMR ( $\delta$, ppm, $400 \mathrm{MHz}$, DMSO-d 6 ): $8.63(\mathrm{~d}, J=8.2 \mathrm{~Hz}, 1 \mathrm{H}), 8.24$ (dd, $2 \mathrm{H}), 8.17-8.09(\mathrm{~m}, 3 \mathrm{H}), 8.01(\mathrm{t}, J=7.8 \mathrm{~Hz}, 1 \mathrm{H}), 7.95(\mathrm{t}, J=7.5 \mathrm{~Hz}, 1 \mathrm{H}), 7.81(\mathrm{t}, J=7.5 \mathrm{~Hz}, 1 \mathrm{H})$, $7.62(\mathrm{t}, J=7.7 \mathrm{~Hz}, 2 \mathrm{H}), 7.36(\mathrm{t}, J=8.7 \mathrm{~Hz}, 2 \mathrm{H}) .{ }^{13} \mathrm{C}$ NMR $\left(\delta, \mathrm{ppm}, 125 \mathrm{MHz}, \mathrm{DMSO}-\mathrm{d}_{6}\right): 186.41$, $163.42,152.72,143.24,142.01,135.07(\mathrm{~d}, J=242.9 \mathrm{~Hz}), 133.25,131.07,130.47,130.06(\mathrm{~d}, J=9.3$ $\mathrm{Hz}), 129.69,129.33,126.70,126.45,124.09,120.29,118.54,116.63(\mathrm{~d}, J=22.1 \mathrm{~Hz}) .{ }^{19} \mathrm{~F}$ NMR $(\delta$, ppm, $376 \mathrm{MHz}$, DMSO-d 6 ): -109.80 . MS (CI): $\mathrm{m} / z 369.2[M+\mathrm{H}]^{+}$. 
6-Benzoyl-3-phenyl-2H-[1,2,4]triazino[2,3-c]quinazolin-2-one (4c): White needle crystals. M.p. 239$245{ }^{\circ} \mathrm{C}$. Yield: $16.5 \% .{ }^{1} \mathrm{H}$ NMR $\left(\delta\right.$, ppm, $\left.400 \mathrm{MHz}, \mathrm{DMSO}-\mathrm{d}_{6}\right): 8.66(\mathrm{~d}, J=8.1 \mathrm{~Hz}, 1 \mathrm{H}), 8.16(\mathrm{~d}, J=$ $7.9 \mathrm{~Hz}, 2 \mathrm{H}), 8.07(\mathrm{t}, J=7.6 \mathrm{~Hz}, 1 \mathrm{H}), 7.98(\mathrm{~d}, J=8.1 \mathrm{~Hz}, 1 \mathrm{H}), 7.89(\mathrm{t}, J=7.5 \mathrm{~Hz}, 1 \mathrm{H}), 7.84-7.73$ $(\mathrm{m}, 3 \mathrm{H}), 7.62(\mathrm{t}, J=7.7 \mathrm{~Hz}, 2 \mathrm{H}), 7.50(\mathrm{t}, J=6.9 \mathrm{~Hz}, 1 \mathrm{H}), 7.39(\mathrm{t}, J=7.6 \mathrm{~Hz}, 2 \mathrm{H}) .{ }^{13} \mathrm{C} \mathrm{NMR}(\delta, \mathrm{ppm}$, $\left.125 \mathrm{MHz}, \mathrm{DMSO}-\mathrm{d}_{6}\right): 187.48,159.88,151.78,150.28,148.04,143.34,136.19,135.82,134.44,131.9$, 131.76, 130.40, 130.31, 129.76, 129.46, 128.78, 128.56, 126.10, 120.62. MS (CI): $m / z 379.2[M+\mathrm{H}]^{+}$.

6-Benzoyl-3-(4-fluorophenyl)-2H-[1,2,4]triazino[2,3-c]quinazolin-2-one (4d): White needle crystals. M.p. $218-223{ }^{\circ} \mathrm{C}$. Yield: $13.7 \% .{ }^{1} \mathrm{H}$ NMR $\left(\delta\right.$, ppm, $\left.400 \mathrm{MHz}, \mathrm{DMSO}-\mathrm{d}_{6}\right): 8.66(\mathrm{~d}, J=8.0 \mathrm{~Hz}, 1 \mathrm{H})$, $8.14(\mathrm{~d}, J=7.7 \mathrm{~Hz}, 2 \mathrm{H}), 8.08(\mathrm{t}, J=7.9 \mathrm{~Hz}, 1 \mathrm{H}), 7.98(\mathrm{~d}, J=8.1 \mathrm{~Hz}, 1 \mathrm{H}), 7.96-7.85(\mathrm{~m}, 3 \mathrm{H}), 7.79$ (t, $J=7.5 \mathrm{~Hz}, 1 \mathrm{H}), 7.61(\mathrm{t}, J=7.8 \mathrm{~Hz}, 2 \mathrm{H}), 7.24(\mathrm{t}, J=8.7 \mathrm{~Hz}, 2 \mathrm{H}) \cdot{ }^{13} \mathrm{C} \mathrm{NMR}(\delta, \mathrm{ppm}, 125 \mathrm{MHz}$, DMSO-d $\left._{6}\right) \delta 187.42,164.43(\mathrm{~d}, J=250.5 \mathrm{~Hz}), 159.84,151.78,149.20,147.95,143.30,136.23$, 135.85, 134.40, $132.05(\mathrm{~d}, J=9.0 \mathrm{~Hz}), 130.41,130.36,129.77,128.57,128.22(\mathrm{~d}, J=2.4 \mathrm{~Hz})$, 126.10, 120.56, 115.99 (d, $J=21.7 \mathrm{~Hz}) .{ }^{19} \mathrm{~F}$ NMR ( $\delta$, ppm, $\left.376 \mathrm{MHz}, \mathrm{DMSO}-\mathrm{d}_{6}\right):-107.99 . \mathrm{MS}(\mathrm{CI})$ : $\mathrm{m} / \mathrm{z} 397.0[\mathrm{M}+\mathrm{H}]^{+}$.

Benzo[4,5]imidazo[1,2-c]quinazolin-6(5H)-one (5a): Beige solid. M.p. $>300{ }^{\circ} \mathrm{C}$. Yield: $33.2 \%,{ }^{1} \mathrm{H}$ $\operatorname{NMR}\left(\delta, \mathrm{ppm}, 500 \mathrm{MHz}, \mathrm{DMSO}-\mathrm{d}_{6}\right): 11.95(\mathrm{~s}, 1 \mathrm{H}), 8.36(\mathrm{~d}, \mathrm{~J}=7.9 \mathrm{~Hz}, 1 \mathrm{H}), 8.31(\mathrm{~d}, \mathrm{~J}=8.0 \mathrm{~Hz}, 1 \mathrm{H})$, $7.86(\mathrm{~d}, \mathrm{~J}=7.9 \mathrm{~Hz}, 1 \mathrm{H}), 7.65(\mathrm{t}, \mathrm{J}=7.7 \mathrm{~Hz}, 1 \mathrm{H}), 7.55-7.29(\mathrm{~m}, 4 \mathrm{H}) .{ }^{13} \mathrm{C} \mathrm{NMR}(\delta, \mathrm{ppm}, 125 \mathrm{MHz}$, DMSO-d $\left._{6}\right): 148.13,146.84,143.98,137.61,132.73,131.09,125.46,124.89,124.10,123.83,119.58$, 116.36, 115.20, 112.25. MS (CI): $m / z 236.1[\mathrm{M}+\mathrm{H}]^{+}$.

Benzoic acid (6): White crystals. M.p. $118-120{ }^{\circ} \mathrm{C}$ (lit. $\left.122.4{ }^{\circ} \mathrm{C}\right) .{ }^{16}$ Yield: 8.1\%. ${ }^{1} \mathrm{H}$ NMR $(\delta$, ppm, 400 MHz, DMSO-d $\left.\mathrm{d}_{6}\right): 12.82(\mathrm{~s}, 1 \mathrm{H}), 7.95(\mathrm{~d}, J=7.2 \mathrm{~Hz}, 2 \mathrm{H}), 7.61(\mathrm{t}, J=7.4 \mathrm{~Hz}, 1 \mathrm{H}), 7.49(\mathrm{t}, J=7.6 \mathrm{~Hz}$, $2 \mathrm{H}) .{ }^{13} \mathrm{C}$ NMR $\left(\delta, \mathrm{ppm}, 125 \mathrm{MHz}, \mathrm{DMSO}-\mathrm{d}_{6}\right): 167.77,133.32,131.23,129.72,129.02 . \mathrm{MS}(\mathrm{CI}): \mathrm{m} / \mathrm{z}$ $121.2[M-\mathrm{H}]^{-}$.

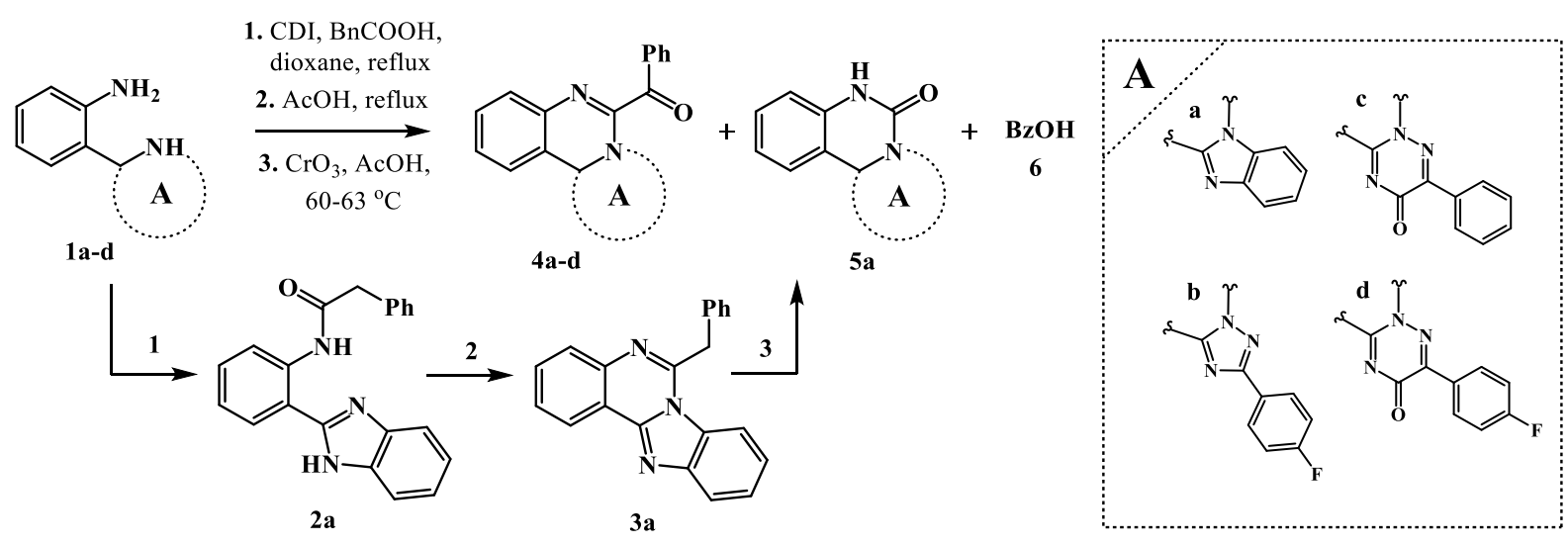

Figure 1. Synthesis and oxidation of some benzyl substituted fused quinazoline derivatives

Oxidation of benzyl substituted fused quinazoline derivatives produced solely one product. Namely, expected corresponding ketones $\mathbf{4 b - d}$, so the chemistry described herein is completely in accordance with the literature. ${ }^{17,18}$ However, in the case of oxidation of 3a, three products were isolated (Figure 1), which were identified as quinazolinone 5a (main product), ketone $\mathbf{4 a}$ and benzoic acid 6. Thus, a novel oxidation process has been uncovered.

Mpreover, a combination of tertiary amine with vicinal carbonyl group was found to be resistant to the oxidation, ${ }^{19}$ assuming the reaction is a Malaprade-like oxidation. ${ }^{20}$ On the other hand, some tetrasubstituted cyclobutanones can undergo a Baeyer-Villiger-like rearrangement to yield the 
corresponding $\gamma$-lactones by chromic acid in a sulfuric acid-acetic acid mixture. ${ }^{21}$ Nevertheless, it does not explain why ketones $\mathbf{4 b}$-d were not oxidized to the corresponding quinazolinones.

A plausible explanation could be that quinazolinone $\mathbf{5 a}$ and benzoic acid $\mathbf{6}$ formations might be due to the less electron-withdrawing effect of imidazole in comparison with 1,2,4-triazole and 1,2,4triazine heterocycles. It let to the supposedly favorable conditions for coordination of the lone pair of the $\mathrm{sp}^{2}$ hybridized quinazoline nitrogen atom on the $\mathrm{Cr}^{\mathrm{VI}}$ atom, resulting in the following degradation of ketone 4a (Figure 2).

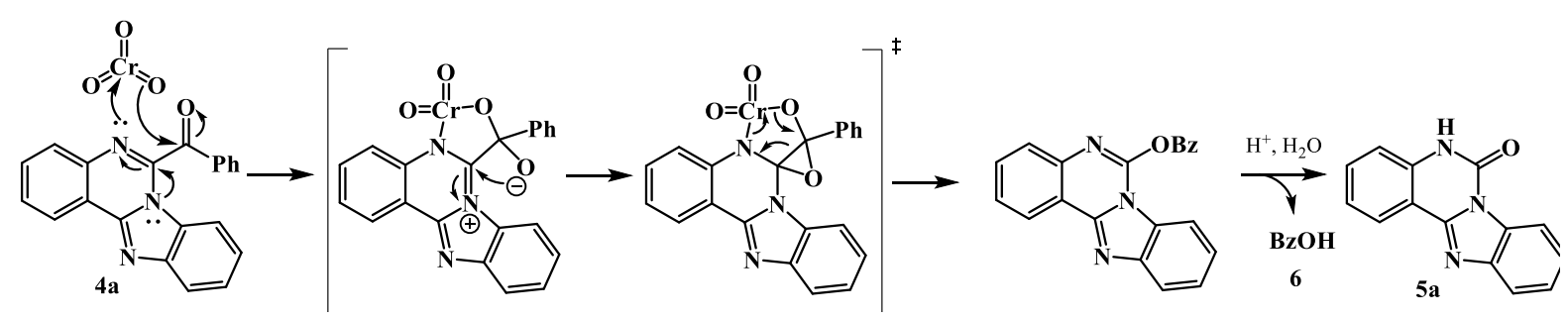

Figure 2. Possible oxidation mechanism of ketone $4 \mathbf{a}$ by $\mathrm{CrO}_{3}$

\section{Conclusion}

In this study, an unexpected interaction mechanism between ketone $\mathbf{4 a}$ and chromium trioxide is disclosed. Besides, three new benzoyl substituted fused quinazoline derivatives $\mathbf{4 b} \mathbf{b}-\mathbf{d}$ were synthesized. The study showed that the level of electron-withdrawing effect of the fused heterocycle played a key role in the reaction. While the stronger one terminated the oxidation at the step of ketone formation, the weaker one yielded quinazolinone as a main product.

\section{Acknowledgements}

The author expresses his gratitude to Prof. Kovalenko S.I. and Dr. Voskoboinik O.Y. for providing some starting compounds and "Enamine Ltd." (Kiev, Ukraine) for financial support.

\section{Supporting Information}

Supporting information accompanies this paper on http://www.acgpubs.org/journal/organic$\underline{\text { communications }}$

\section{ORCID}

Dmytro Kravtsov: 0000-0001-6880-2518

\section{References}

[1] Kuarm, B. S.; Reddy, Y. T.; Madhav, J. V.; Crooks, P. A.; Rajitha, B. 3-[Benzimidazo- and 3[benzothiadiazoleimidazo-(1,2-c)quinazolin-5-yl]-2H-chromene-2-ones as potent antimicrobial agents. Bioorg. Med. Chem. Lett. 2011, 21, 524-527.

[2] Habib, O. M. O.; Hassan H. M.; El-Mekabaty, A. Novel quinazolinone derivatives: synthesis and antimicrobial activity. Med. Chem. Res. 2013, 22, 507-519.

[3] El-Sayed R.; Wasfy, A. A.F.; Aly, A. A. Synthesis of novel heterocycles with antimicrobial and surface activity. J. Het. Chem. 2005, 42, 125-130.

[4] Rohini, R.; Shanker, K.; Reddy, P. M.; Ho, Y.-P. Ravinder, V. Mono and bis-6-arylbenzimidazo[1,2c]quinazolines: A new class of antimicrobial agents. Eur. J. Med. Chem. 2009, 44, 3330-3339.

[5] Rohini, R.; Shanker, K.; Reddy, P. M.; Ravinder, V. Synthesis and antimicrobial activities of a new class of 6-arylbenzimidazo[1,2-c]quinazolines. J. Braz. Chem. Soc. 2010, 21(1), 49-57.

[6] Vostrova, L. N.; Voronina, T. A.; Karaseva, T. L.; Gernega, S. A.; Ivanov, E. I.; Kirichenko, A. M.; Totrova, M. Yu. Synthesis and anticonvulsant activity of benzimidazo[1,2-c]quinazolines. Pharm. Chem. J. 1986, 6, 404-406. 
[7] Bahekar, R. H.; Rao, A. R. R. Bronchodilation and structure-activity relationship studies on new 6substituted benzimidazo[1,2-c]quinazolines. Arzneim.-Forsch./Drug Res. 2000, 50 (2), 712-716.

[8] Fieser, L. F.; Szmuszkovicz, J. Synthesis of keto acids by chromic anhydride oxidation of tertiary alcohols. $J$. Am. Chem. Soc. 1948, 70, 3352-3355.

[9] Harmsla, W. M.; Eisenbraun, E. J. Chromic acid oxidation of indans and hydrindacenes. Synthesis of 1,1dimethylindene. Org. Prep. Proced. Int. 1972, 4 (2), 67-72.

[10] Smith, L. I.; Webster, I. M. The reaction between quinones and sodium enolates. V. 2,3Dimethylnaphthoquinone and sodium malonic ester. J. Am. Chem. Soc. 1937, 59, 662-667.

[11] Hein, D. W.; Alheim, R. J.; Leavitt, J. J. The use of polyphosphoric acid in the synthesis of 2-aryl- and 2alkyl-substituted benzimidazoles, benzoxazoles and benzothiazoles. J. Am. Chem. Soc. 1957, 79 (2), $427-$ 429.

[12] Kholodnyak, S. V.; Schabelnyk, K. P.; Zhernova, G. O.; Sergeieva, T. Yu.; Ivchuk, V. V.; Voskoboynik, O. Yu.; Kovalenko, S. I.; Trzhetsinskii, S. D.; Okovytyy, S. I.; Shishkina, S. V. Hydrolytic cleavage of the pyrimidine ring in 2-aryl-(1,2,4)triazole(1,5-c)quinazolines: physico-chemical properties and the hypoglycemic activity of the compounds synthesized. News of Pharmacy 2015, 3, 9-17.

[13] Sergeieva, T. Yu.; Voskoboynik, O. Yu.; Okovytyy, S. I.; Kovalenko, S. I; Shishkina, S. V.; Shishkin, O. V.; Leszczynski, J. Hydrazinolysis of 3-R-[1,2,4]triazino[2,3-c]quinazolin-2-ones. Synthetic and theoretical aspects. J. Phys. Chem. A 2014, 118, 1895-1905

[14] Linhua, X.; Tingfang, L.; Lianhui, W.; Xiuling, C. Rh(III)-catalyzed one-pot synthesis of benzimidazoquinazolines via $\mathrm{C}-\mathrm{H}$ amidation-cyclization of $\mathrm{N}$-LG-2-phenylbenzoimidazoles. J. Org. Chem. 2019, 84 (2), 560-567.

[15] Ambethkar, S.; Kalaiselvi, M.; Ramamoorthy, J.; Padmini, V. I ${ }_{2}$-Catalyzed oxidative cross-coupling reaction of methyl ketones and 2-(2-aminophenyl) benzimidazole: facile access to benzimidazo[1,2-c]quinazoline. ACS Omega 2018, 3, 5021-5028.

[16] O'Neil, M. J.; Smith, A.; Heckelman, P. E. The Merck index : an encyclopedia of chemicals, drugs, and biological, Merck, 1996.

[17] Takashi Kishimoto; Yasuo Yamada; Takao Iwasa; Michihiko Matsuda; Renpei Hatano. Triazole compounds and pest control agent. United Stated Patent 1999, US 005892048A, Example 7.

[18] Christoph Braun; Haruko Sawada; Helene Lachaise; Stephane Brunet; Philippe Rinolfi; Christophe Dubost; Pierre-Yves Coqueron; Simon Maechling; Anne-Sophie Rebstock; Ulrike Wachendorff-Neumann. Fungicidal 4-substituted-3-\{phenyl[(heterocyclylmethoxy)imino]methyl \}-1,2,4-oxadizol-5(4H)-one derivatives. World Intellectual Property Organization | International Application Published Under the Patent Cooperation Treaty 2013, WO 2013/037717 A1, 140.

[19] Nicolet, B. H.; Shinn, L. A. The action of periodic acid on $\alpha$-amino alcohols. J. Am. Chem. Soc. 1939, 61, 1615.

[20] Wang, Z. Malaprade Reaction Comprehensive Organic Name Reactions and Reagents, 2010.

[21] Jeanne-Carlier, R.; Bourelle-Wargnier, F. Synthese et oxydation chromique de cyclobutanones tetrasubstituees. Bull. Chim. Soc. Fr. 1976, 1-2 (2), 297-300.

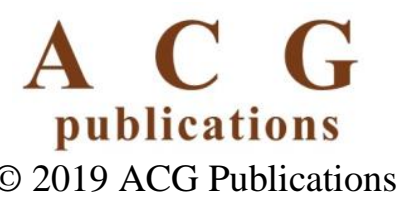

